\title{
Do MRI features at baseline predict radiographic joint space narrowing in the medial compartment of the osteoarthritic knee 2 years later?
}

\author{
Ruby Madan-Sharma • Margreet Kloppenburg • \\ Peter R. Kornaat • Stella A. Botha-Scheepers • \\ Marie-Pierre Hellio Le Graverand • \\ Johannes L. Bloem • Iain Watt
}

Received: 14 August 2007 /Revised: 4 January 2008/Accepted: 7 April 2008 /Published online: 20 June 2008

(C) The Author(s) 2008

\begin{abstract}
Purpose The purpose of the study was to relate magnetic resonance imaging (MRI) features at baseline with radiographically determined joint space narrowing (JSN) in the medial compartment of the knee after 2 years in a group of patients with symptomatic osteoarthritis at multiple joint sites. Materials and methods MRI of the knee and standardized radiographs were obtained at baseline and after 2 years in 186 patients ( $81 \%$ female; aged $43-76$ years; mean 60 years). MRI was analyzed for bone marrow lesions, cysts, osteophytes, hyaline cartilage defects, joint effusion, and meniscal pathology in the medial compartment. Radiographs were scored semiquantitatively for JSN in the medial tibiofemoral joint using the Osteoarthritis Research Society International (OARSI) atlas. Radiological progression was defined as $\geq 1$ grade increase. Associations between baseline magnetic resonance (MR) parameters and subsequent radiographic JSN changes were assessed
\end{abstract}

Grant supporter: Pfizer Groton, Groton, CT, USA

Pfizer Inc., Groton, CT, USA provided financial support for this work. However, the authors are not employees of Pfizer Inc., retaining full control of inclusion of all data and information that might present a conflict of interest.

R. Madan-Sharma $(\bowtie) \cdot$ P. R. Kornaat · J. L. Bloem • I. Watt Department of Radiology, Leiden University Medical Center, C3-S, P.O. Box 9600, 2300 RC Leiden, Netherlands

e-mail: R.Sharma@lumc.nl

M. Kloppenburg $\cdot$ S. A. Botha-Scheepers

Department of Rheumatology, Leiden University Medical Center, Leiden, Netherlands

M.-P. H. Le Graverand

Pfizer Groton,

Groton, CT, USA using logistic regression. Relative risk (RR) was then calculated.

Results Radiographic progression of JSN was observed in $17(9.1 \%)$ of 186 patients. Eleven patients had a Kellgren and Lawrence $(\mathrm{KL})$ score of $\geq 2$. A significant association was observed between all patients and meniscal tears (RR 3.57; confidence interval (CI) 1.08-10.0) and meniscal subluxation (RR 2.73; CI 1.20-5.41), between $\mathrm{KL}<2$ and meniscal subluxation (RR 11.3; CI 2.49-29.49) and $\mathrm{KL} \geq 2$ and meniscus tears (RR 8.91; CI 1.13-22.84) and radiographic JSN 2 years later. Follow-up MR in 15 of 17 patients with progressive JSN showed only new meniscal abnormalities and no progression of cartilage loss.

Conclusion Meniscal pathology (tears and/or meniscal subluxation) was the only MRI parameter to be associated with subsequent radiographic progression of JSN in the medial tibiofemoral compartment on a radiograph 2 years later, as assessed by the OARSI score.

Keywords Knee MRI · Osteoarthritis · Meniscus pathology

\section{Introduction}

Knee osteoarthritis (KOA), characterized by cartilage and bony alterations, is a progressive disease accompanied by pain and stiffness and is one of the major causes of musculoskeletal disability in the industrialized world with an increasing prevalence related to an aging population [1].

Currently, not only is the pathogenesis and etiology of osteoarthritis (OA) not fully understood but also no approved disease modifying OA drugs are available. To date, it is not clear which parameters predict progression of 
OA. Thus, it is important to find parameters by which the more rapidly progressing patients can be identified to focus upon them in trials of the novel therapies currently being developed and eventually to treat this population.

At present, plain film radiography is the method of choice to grade $\mathrm{OA}$ by assessing structural damage. This technique permits assessment of osteophytes, subchondral bone cysts, sclerosis, and joint space width (JSW). Changes in JSW, the gold standard for the evaluation of $\mathrm{OA}$ progression in clinical trials, are related to various abnormalities including cartilage loss and meniscal subluxation or extrusion from the joint line [2]. However, JSW measurement does not permit detection of structural changes in nonopaque tissues nor does it constitute an efficient means of monitoring the progression of $\mathrm{OA}$ in daily practice [3, 4]. Further, radiographic measurement of JSN in the knee is reliable only in the medial compartment [5].

Recently, magnetic resonance (MRI) imaging data have fueled the debate on the relevance of radiographic JSW as a single measure of biological severity in knee OA since knee $\mathrm{OA}$ is panarticular and mild to moderate joint space loss may result from changes in other structures such as the menisci [6].

The question arises whether prognostic magnetic resonance (MR) features can be determined which can predict JSN over a period of 2 years as seen on a standard knee radiograph. Different structural abnormalities such as joint effusion and synovial thickening [7], osteophytes [8,9], cartilage lesions [10], and patellar cartilage volume [11] have been attributed to the progression of KOA. Bone marrow lesions (BML) also have been associated with progression of knee OA [12] but BML and cysts may change over time and their prognostic significance remains uncertain [13].

The purpose of the present study was to relate MRI parameters at baseline with radiographic progression of knee OA as assessed by JSN after 2 years.

\section{Methods and materials}

\section{Patients}

The present study is part of the ongoing Genetics, Osteoarthritis, and Progression (GARP) study [14]. The primary goal of GARP is to determine risk factors for OA development and disease progression. Probands, aged between 40 and 70 years, and their siblings $(n=382)$, with symptomatic $\mathrm{OA}$ at multiple sites were eligible after giving informed consent. Sibling pairs $(n=210)$ with at least one subject having symptomatic hip OA or knee OA (but not radiological end-stage disease defined as a Kellgren and Lawrence (KL) score of 4 [15]) were followed for 2 years. Of the 210 patients at baseline, 186 completed the 2-year follow-up with complete data and were thus included.
Median age was 60.2 years; 150 were female, and mean body mass index (BMI) was $26.5 \mathrm{~kg} / \mathrm{m}^{2}$ (Table 1). Of the included patients, 74 had definite knee OA as defined by a KL score of 2 or more. Our institution's medical ethical review board approved the study.

\section{Diagnosis of osteoarthritis}

Subjects were included with symptomatic OA at two or more of the following joints sites: hand, spine (cervical or lumbar), knee, or hip defined by the presence of radiographic OA in any of the four joints groups, or for the hands the presence of two or more Heberden nodes, Bouchard nodes, or squaring of at least one first carpometacarpal (CMCJ1) joint on physical examination.

Symptomatic OA in the knee and hip was defined according to the American College of Rheumatology recommendations for knee and hip OA $[16,17]$. Knee OA was defined as pain or stiffness for most days of the preceding month and osteophytes at the joint margins of the tibiofemoral joint (X-ray spurs). Hip OA was defined as pain or stiffness in the groin and hip region on most days of the preceding month in addition to femoral or acetabular osteophytes or axial joint space narrowing on radiography. Prosthetic joints in the hips or knees as a result of end-stage $\mathrm{OA}$ were defined as $\mathrm{OA}$ in that particular joint.

\section{Radiograph acquisition}

The standardized, nonfluoroscopic, fixed-flexion protocol was used in a single center to obtain posteroanterior weightbearing radiographs of the knee at baseline and after 24 months [18]. Uniform anatomical alignment of the knees was facilitated by the use of a specifically designed positioning frame (Synaflexer ${ }^{\circledR}$, San Francisco, USA) [18] that places the patient's feet in $5^{\circ}$ of external rotation, the knees and thighs in contact with the cassette and coplanar

Table 1 Demographics of 186 patients with OA at multiple sites and characteristics of imaged knees in these subjects

Characteristics

Age (years), median (range)

Sex (female), $N(\%)$

BMI $\left(\mathrm{kg} / \mathrm{m}^{2}\right)$, median (range)

Knee pain ${ }^{\mathrm{a}}$, N (\%)

Symptomatic knee OA, $N(\%)$

KL score, no. (\%)

$>1$

$=1$

$55(29.6)$

$=0$

$57(30.6)$

$B M I$ Body mass index, $K L$ Kellgren-Lawrence score, 0-3

${ }^{\text {a }}$ Pain on most days of the prior months in the imaged knee as described by ACR 
with the hips and tips of the great toes, resulting in a fixed knee angulation of approximately $20^{\circ}$ flexion. The X-ray tube was angled to point $10^{\circ}$ downwards and the knees were exposed to the X-ray beam centered at the joint line. Radiographic severity of knee OA at baseline was scored according to the KL score [15]. The intraclass correlation coefficient for reproducibility was 0.92 [14].

\section{Radiographic progression}

Baseline and 2-year knee radiographs were scored for medial JSW on a scale of 0 (normal)-3 (total JSN) with help of the Osteoarthritis Research Society International (OARSI) atlas $[19,20]$ by two experienced readers (respectively 3 and 30 years of experience) reading in consensus. In cases of disagreement, the lower, more conservative score was adopted. An increase of at least 1 grade between baseline and follow-up was considered to be radiographic progression. The observers were blinded to clinical information such as age, gender, and time sequence.

\section{MRI acquisition}

Knees were imaged using a dedicated knee coil in a 1.5-T magnet (Philips Medical Systems, Best, the Netherlands). Each examination consisted of the following sequences, optimized for software available at time of inclusion from 2002 to 2005: (1) Coronal proton density (PD) and T2weighted dual spin echo (SE) sequence (repetition time (TR) of 2,200, an echo time (TE) of 20/80, 5-mm slice thickness, $0.5-\mathrm{mm}$ intersection gap, 160-mm field of view (FOV), and $205 \times 256$ acquisition matrix); (2) sagittal PD and T2-weighted dual SE images (TR 2,200, TE 20/80, 4-mm slice thickness, 0.4-mm intersection gap, 160-mm FOV, $205 \times 256$ acquisition matrix); (3) sagittal 3D T1-weighted spoiled gradient echo (GE) frequency-selective fat-suppressed images (TR 46, TE 2.5 , flip angle $40^{\circ}, 3.0-\mathrm{mm}$ slice thickness, slice overlap $1.5 \mathrm{~mm}$, no gap, 180 -mm FOV, $205 \times 256$ acquisition matrix); (4) axial proton density and T2-weighted turbo SE fatsuppressed images (TR 2,500, TE 7.1/40, echo train length 6, 2-mm slice thickness, no gap, 180-mm FOV, 205×256 acquisition matrix). The total acquisition time (including the initial survey sequence) was $30 \mathrm{~min}$.

MR images at baseline of the knee were performed successfully in 205 of 208 patients. One patient was excluded due to claustrophobia, and in two others image quality was inadequate due to motion artifacts.

\section{MRI interpretation}

All MR images were analyzed by means of consensus between three readers. During the assessment, the readers were blinded to radiographic results, patient symptoms, patient age, and other clinical data. A published knee OA scoring system (KOSS) was used to assess osteoarthritic defects [21]. The MR images of patients including a 2-year follow-up MRI were assessed simultaneously using the $\Delta$ KOSS [21].

Cartilaginous defects and BML were assigned to any of the following anatomical locations: the crista patellae, medial or lateral patellar facet, the medial or lateral trochlear facets, the medial or lateral femoral condyle, and the medial or lateral tibial plateau. The medial and lateral menisci were reviewed for the presence of meniscal tears and/or subluxation out of the joint line.

Cartilaginous defects were graded as diffuse or focal. The coronal and sagittal SE images and sagittal GE images were used to assess the tibiofemoral cartilage. Axial turbo SE images and sagittal GE and SE images were used to assess patellofemoral cartilage. The surface extent of a diffuse or focal cartilaginous defect was classified by its maximal diameter and was graded as follows: grade 0 , absent; grade 1, minimal $(<5 \mathrm{~mm})$; grade 2 , moderate $(5-$ $10 \mathrm{~mm})$; grade 3, severe $(>10 \mathrm{~mm})$. The depth of a cartilaginous defect was graded using a modification of the Yulish classification [22]: grade 0, absent; grade 1, $<50 \%$ reduction of cartilage thickness; grade $2,>50 \%$ reduction of cartilage thickness; grade 3, full thickness or nearly full thickness cartilage defect.

A BML was defined as an ill-defined area of increased signal intensity on $\mathrm{T} 2$-weighted images in the subchondral bone, extending away from an articular surface or at places where traction changes occur [23]. The lesions were graded as follows: grade 0, absent; grade 1, minimal $(<5 \mathrm{~mm})$; grade 2, moderate $(5-2 \mathrm{~cm})$; grade 3 , severe $(>2 \mathrm{~cm})$.

A meniscal tear was defined as a region of intermediate signal intensity on PD-weighted images within the meniscus, communicating with its superior and/or inferior surfaces or inner margin, on more than one slice [24]. The meniscal roots were examined also.

Meniscal subluxation was defined as protrusion over the edge of the tibial plateau seen at the level of the body of the meniscus on coronal PD-weighted images and was graded as follows: grade 0 , absent; grade 1 , minimal $(<1 / 3$ width of the meniscus bulging); grade 2 , moderate $(1 / 3-2 / 3$ meniscal width involved); grade 3 , severe $(>2 / 3$ meniscal width involved).

Osteophytes were defined as focal bony excrescences, seen on axial, sagittal, or coronal images, extending from a cortical surface. Osteophytes were further specified as being marginal, intercondylar, or central. Osteophytes were assessed using the following scale: grade 0 , absent; grade 1 , minimal $(<3 \mathrm{~mm})$; grade 2 , moderate $(3-5 \mathrm{~mm})$; grade 3 , severe $(>5 \mathrm{~mm})[25]$.

Subchondral cysts were defined as well-defined foci of high signal intensity on $\mathrm{T} 2$-weighted images, in the 
cancellous bone underlying the joint cartilage. Their greatest dimension was measured and they were graded as follows: grade 0 , absent; grade 1 , minimal $(<3 \mathrm{~mm})$; grade 2 , moderate $(3-5 \mathrm{~mm})$; grade 3 , severe $(>5 \mathrm{~mm})$.

The presence of joint effusion was evaluated on T2weighted coronal, sagittal, and axial sequences. A joint effusion was assumed to be absent when only a small physiological sliver of synovial fluid was observed. A small effusion was scored as present when a small amount of fluid distended one or two of the joint recesses (suprapatellar pouch, medial or lateral patellar recess, dorsal tibiofemoral joint space, popliteal tendon sheath, recesses surrounding the cruciate ligaments, meniscosynovial recesses), a moderate effusion when more than two joint recesses were partially distended, and massive effusion when full, marked distention of all the joint recesses was present.

\section{Statistical analysis}

Logistic regression analysis was used to calculate odds ratios (ORs) and 95\% confidence intervals (CIs) to determine a relation between MR parameters seen at baseline and JSN on radiographs 2 years later for the entire population and separately for the $\mathrm{KL}<2$ group and $\mathrm{KL} \geq 2$ of patients. The ORs were adjusted for sex, age, BMI, and family effect using STATA 8.0 for Windows (STATA Corp, TX, USA). These ORs were then converted to relative risks (RR) and $95 \%$ CI were calculated also using the formula described by Zhang and $\mathrm{Yu}$ [26].

In patients showing JSN, the baseline grades were subtracted from the scores 2 years later. For cartilage, change in either diffuse thinning or focal thinning on either the femur or tibia facet was considered to be progression. This was also the case for osteophytes, BML, and subchondral cysts. For meniscal tears and subluxation, progression was considered to be a new tear or extension of the original tear into any of the other compartments (anterior, body, or posterior). This was analyzed also by subtracting the baseline score from the score 2 years later.

\section{Results}

The majority (112 or $60 \%)$ of patients had no significant features of knee $\mathrm{OA}(\mathrm{KL}=0$ or 1$)$, (Table 1$)$. At 2 years, radiographic progression of JSN occurred in 17 patients $(9 \%)$. In the knees with radiographic OA (KL $\geq 2), 11$ of 74 patients $(15 \%)$ had progressive JSN, whereas six of 112 (5.4\%) progressed in the $\mathrm{KL}<1$ group.

In the entire patient population, a significant association was found between meniscal tears (crude RR 4.1; crude CI 1.3-13.1; adjusted RR 3.57; adjusted CI 1.08-10.0) and meniscal subluxation (crude RR 3.5; crude CI 1.3-9.8; adjusted RR 2.73; adjusted CI 1.20-5.41) with progression of radiographic JSN (Table 2). No association was seen between the progression of JSN in the entire study population over 2 years and BML, cartilage loss, and joint effusion seen at baseline. A trend for an association was seen with subchondral cysts.

In the $\mathrm{KL}<2$ group, a definite association occurred between meniscal subluxation and radiographic progression of JSN 2 years later (crude RR 7.85; crude CI 1.56-24.86; adjusted RR 11.3; adjusted CI 2.49-29.49). An example is shown in Fig. 1a-d. A trend was seen for BML and cartilage loss, but no other association was shown between the progression of JSN and any other MR parameter (Table 2).

An association was seen also in the $K L \geq 2$ group between meniscal tears and radiographic progression of JSN 2 years later (crude RR 8.91; crude CI 1.1-22.8;

Table 2 The association between MRI characteristics in the medial tibiofemoral compartment and radiographic progression after 2 years in all subjects and only in patients with $\mathrm{KL} \geq 2$ at baseline separately

\begin{tabular}{llll}
\hline Prognostic factor & \multicolumn{2}{l}{ Adjusted $^{\mathrm{a} R}$ RR $(95 \% \mathrm{CI})$} & \\
\cline { 2 - 4 } & All subjects & $\mathrm{KL}<2$ & $\mathrm{KL} \geq 2$ \\
& $N=186$ & $N=112$ & $\mathrm{~N}=74$ \\
\hline Bone marrow edema & $1.8(0.51-5.17)$ & $4.97(0.31-18.15)$ & $0.9(0.18-3.0)$ \\
Cysts & $2.7(0.8-6.4)$ & $1.66(0.23-8.35)$ & $1.6(0.5-4.0)$ \\
Cartilage loss & $1.7(0.4-6.7)$ & $2.72(0.37-12.79)$ & $3.0(0.5-9.6)$ \\
Osteophytes & $2.1(0.6-6.4)$ & $0.52(0.05-3.26)$ & - \\
Meniscal tears & $3.57(1.1-10.0)$ & $1.19(0.14-6.92)$ & $8.91(1.1-22.8)$ \\
Meniscal subluxation & $2.73(1.2-5.4)$ & $11.27(2.49-29.49)$ & $1.70(0.6-3.7)$ \\
Joint effusion & $1.35(0.7-2.4)$ & $2.41(0.51-8.63)$ & $0.6(0.2-1.8)$ \\
\hline
\end{tabular}

$R R$ Relative risk, 95\% CI 95\% confidence intervals, $K L$ Kellgren and Lawrence

${ }^{a}$ Adjustment for age, sex, BMI, and family effect 

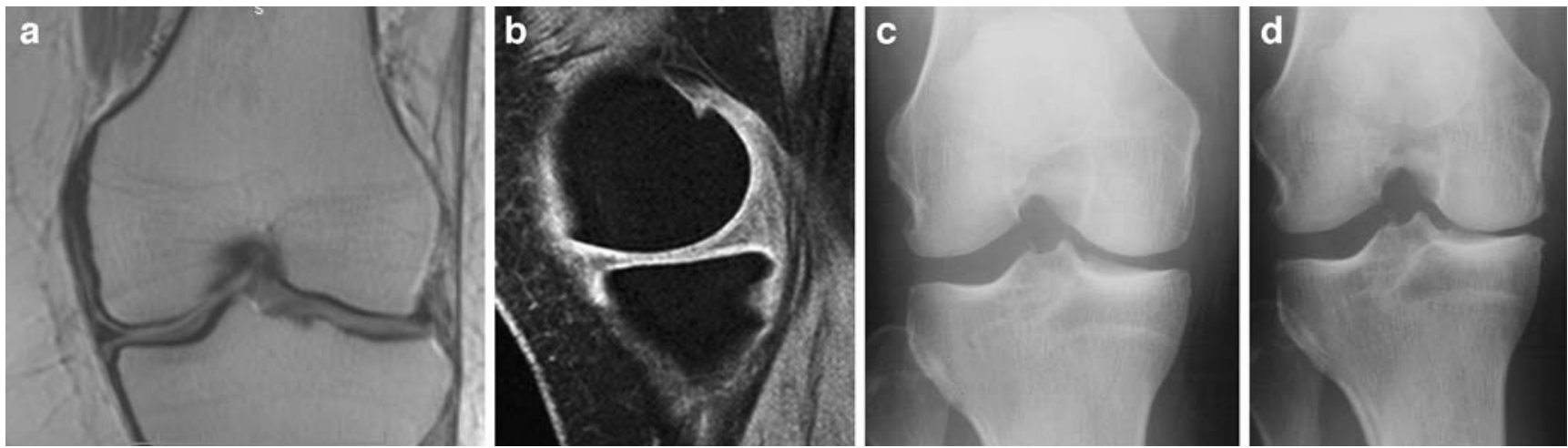

Fig. 1 Coronal PD dual SE images medial meniscus subluxation (a) with a sagittal 3D T1-weighted spoiled gradient echo frequencyselective fat-suppressed image of normal hyaline cartilage (b) with X-

rays of subsequent joint space narrowing in the medial compartment after 2 years (d) compared to baseline (c) in the $\mathrm{KL}<2$ group

adjusted RR 8.91; adjusted CI 1.13-22.84). This is illustrated in Fig. 2a-d. A trend was seen for cartilage loss. However, no association was shown between the progression of JSN and BML, subchondral cysts, and effusion seen at baseline. The association between osteophytes and progression could not be assessed as, by definition, all patients with $\mathrm{KL} \geq 2$ had osteophytes at baseline.

Table 3 shows the MR changes in the knees with JSN over 2 years. Of 17 patients, 15 showed JSN 2 years later on X-ray (two patients did not have the follow-up MRI scans). While no loss of hyaline cartilage was observed in these 15 knees, four new meniscal tears and three meniscal subluxations were observed.

\section{Discussion}

Only meniscal subluxation and meniscal tears in particular were significant in predicting radiographic JSN in the medial compartment of the knee 2 years later in the entire study population. In the $\mathrm{KL} \geq 2$ subpopulation, the effect of meniscal subluxation was not significant as it was in the $\mathrm{KL}<2$ group, but the presence of meniscal tears was still found to be a significant risk factor. Hunter et al. [27] have demonstrated also that alterations in the menisci lead to joint space variance and loss of width. The presence of a tear alters weight-bearing forces and causes cartilage water redistribution and/or actual degeneration and thus a reduction in JSW [28]. In the KL $<2$ group, meniscal subluxation was a major cause for radiographic JSN in the medial compartment. In a much smaller study population of $30 \mathrm{OA}$ patients, Adams et al. [6] also have shown that meniscal subluxation on MR imaging is associated with JSN on Xrays of OA but in a less disease-advanced population and over a shorter time period of only 1 year. However, in the present series, it is unclear why meniscal subluxation should be a risk factor for $\mathrm{KL}<2$ and not in the $\mathrm{KL} \geq 2$ patients. Possible explanations include laxity of the capsule and/or medial collateral ligament early in the evolution of OA. Others include extrusion from the tibiofemoral joint line secondary to marginal osteophyte formation as ob-

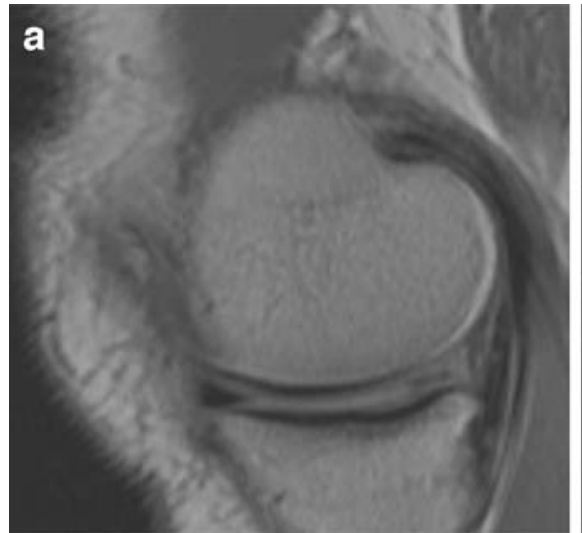

Fig. 2 Sagittal PD-weighted MR image shows a partial maceration of the posterior horn of medial meniscus (a) with a sagittal 3D T1weighted spoiled gradient echo frequency-selective fat-suppressed
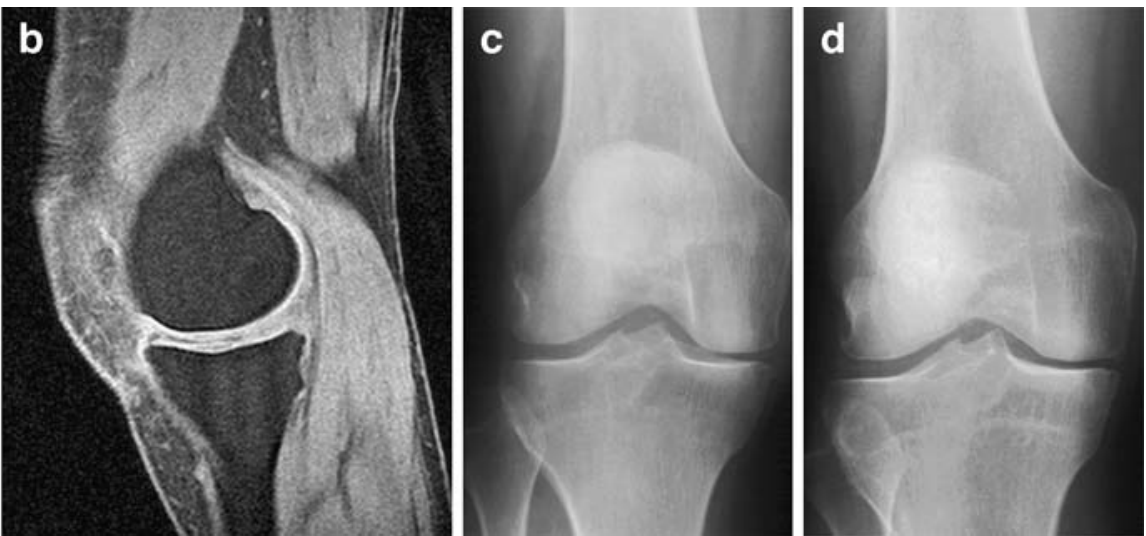

image of minimal hyaline cartilage loss (b) with X-rays of subsequent joint space narrowing in the medial compartment after 2 years (d) compared to baseline (c) in the $\mathrm{KL} \geq 2$ group 
Table 3 The MR changes over 2 years in the medial femoral tibial compartment observed in 15 out of 17 patients with progressive JSN on radiographs for each prognostic factor

Two patients were lost to follow-up.

\begin{tabular}{llll}
\hline Prognostic Factor & MR Change & & \\
\cline { 2 - 4 } & No change & Larger & Smaller \\
\hline Bone marrow edema & 13 & 0 & 2 \\
Subchondral cysts & 11 & 2 & 2 \\
Cartilage loss & 14 & 0 & 1 \\
Osteophyte & 11 & 3 & 1 \\
Meniscus tear & 10 & 4 & 1 \\
Meniscus subluxation & 12 & 3 & 0 \\
Joint effusion & 12 & 1 & 3 \\
\hline
\end{tabular}

served in a cross-sectional study (OR 4.89; CI 1.59-15.02) [29]. Also, it is possible that in OA subluxation is a precursor to a degenerative meniscal tear. Another possibility is of increased mobility secondary to root tears. These are tears originating at the root of the meniscus and therefore allowing extrusion of the meniscus by increased mobility $[6,30]$. These were not detected in this study.

The obvious question arises as to whether the JSN observed in our patients is a real finding. The follow-up MR scans 2 years later confirmed that meniscal lesions were the main features to have changed. Hyaline cartilage loss was not the etiology (Table 3). In some cases, BML, subchondral cysts, and effusion improved. However, these are known to be dynamic processes on MR imaging [13]. However, the apparent improvement in osteophyte formation seen in one patient may be an artifact of projection. The observation of less cartilage loss in a patient 2 years later could indeed be spontaneous repair as is seen in animal models [31] or it may just be an artifact.

Others have shown that hyaline cartilage pathology at baseline does seem to predict JSN, but in our study the association was not significant even though a trend was seen. Cartilage defects do not seem to account for substantial JSN [32, 33].

The underlying rationale for investigating the use of MR imaging in predicting progression in radiographic JSN was to enable identification of those subjects with a likelihood of rapid progression of JSN for selection in clinical trials with novel treatments. In most studies, plain radiographs remain the "gold standard" for assessing knee OA and MRI is seen as an expensive adjunct. However, was a feature to be seen on a baseline MRI that did predict subsequent cartilage loss, justification of an MR image would increase and perhaps also permit greater understanding of the underlying pathophysiology of OA. The literature suggests that subsequent JSN would be primarily related to cartilage abnormalities [5]. This was not the case in our population in whom the inclusion criteria specified an earlier or less severe grade of knee OA than other studies [12]. Further, even though cartilage defects were seen at baseline, they did not appear to be the primary reason for subsequent JSN, again questioning how much apparent JSN in knee OA really represents hyaline cartilage pathology.

The results in the present study with regard to BML and progression of $\mathrm{OA}$ are not in agreement with the findings of others [34]. In the Boston Osteoarthritis of the Knee Study (BOKS), the finding of BML pattern carried seven times higher risk for progression of OA [34]. This was not confirmed in the present study (RR 1.8; CI 0.51-5.17). However, the BOKS [12] comprised patients with established KOA, whereas in the present study the population consisted of OA patients with generalized OA, where not all patients had established knee OA. Also, the median BMI of our study population was lower than in the BOKS. In addition, Kornaat et al. [13] have reported that BML patterns fluctuate significantly in most OA knees in that study over time (i.e., one-third improving and another third worsening), indicating that BML is a dynamic phenomenon. Taken together, the prognostic value of a BML pattern for progression of OA seems not to be fully established. Currently, the role of BML patterns in early OA remains ambiguous.

In conclusion, in the present MR report, meniscal pathology (tears and/or meniscal subluxation) was the only MR parameter to be associated with subsequent radiographic progression of JSN in the medial tibiofemoral compartment seen on a radiograph 2 years later as assessed by the OARSI score. These data indicate that the predictive significance of other MR parameters in OA of the knee remain ambiguous in a time span of 2 years in a population with mild knee OA.

This study has some limitations which need to be addressed. The first one is the small number of progressors in this sample population. Secondly, the patient population comprises of patients with generalized OA and only $40 \%$ presented with knee OA which may account for the small number of progressors in this study. Thirdly, as this study is in an early cohort of OA patients, the follow-up period of 2 years may be too short to observe changes. Therefore, it would be interesting to look at early OA patients with a longer followup period of 5 years to assess the consequences of meniscal lesions, cartilage lesions and BML, and cysts. 
Open Access This article is distributed under the terms of the Creative Commons Attribution Noncommercial License which permits any noncommercial use, distribution, and reproduction in any medium, provided the original author(s) and source are credited.

\section{References}

1. Felson DT. An update on the pathogenesis and epidemiology of osteoarthritis. Radiol Clin North Am 2004; 42(1): 1-9, v.

2. Felson DT, Zhang Y, Hannan MT et al. The incidence and natural history of knee osteoarthritis in the elderly. The Framingham Osteoarthritis Study. Arthritis Rheum 1995; 38(10): 1500-1505.

3. Brandt KD, Fife RS, Braunstein EM, Katz B. Radiographic grading of the severity of knee osteoarthritis: relation of the Kellgren and Lawrence grade to a grade based on joint space narrowing, and correlation with arthroscopic evidence of articular cartilage degeneration. Arthritis Rheum 1991; 34(11): 1381-1386.

4. Hodler J, Resnick D. Current status of imaging of articular cartilage. Skeletal Radiol 1996; 25(8): 703-709.

5. Buckland-Wright JC, Macfarlane DG, Lynch JA, Jasani MK, Bradshaw CR. Joint space width measures cartilage thickness in osteoarthritis of the knee: high resolution plain film and double contrast macroradiographic investigation. Ann Rheum Dis 1995; 54(4): 263-268.

6. Adams JG, McAlindon T, Dimasi M, Carey J, Eustace S. Contribution of meniscal extrusion and cartilage loss to joint space narrowing in osteoarthritis. Clin Radiol 1999; 54(8): 502-506.

7. Hill CL, Gale DG, Chaisson CE et al. Knee effusions, popliteal cysts, and synovial thickening: association with knee pain in osteoarthritis. J Rheumatol 2001; 28(6): 1330-1337.

8. Boegard T, Rudling O, Petersson IF, Jonsson K. Correlation between radiographically diagnosed osteophytes and magnetic resonance detected cartilage defects in the tibiofemoral joint. Ann Rheum Dis 1998; 57(7): 401-407.

9. Boegard T. Radiography and bone scintigraphy in osteoarthritis of the knee-comparison with MR imaging. Acta Radiol Suppl 1998; 418: 7-37.

10. Link TM, Steinbach LS, Ghosh S et al. Osteoarthritis: MR imaging findings in different stages of disease and correlation with clinical findings. Radiology 2003; 226(2): 373-381.

11. Hunter DJ, March L, Sambrook PN. The association of cartilage volume with knee pain. Osteoarthritis Cartilage 2003; 11(10): $725-729$.

12. Felson DT, Chaisson CE, Hill CL et al. The association of bone marrow lesions with pain in knee osteoarthritis. Ann Intern Med 2001; 134(7): 541-549.

13. Kornaat PR, Kloppenburg M, Sharma $R$ et al. Bone marrow edema-like lesions change in volume in the majority of patients with osteoarthritis; associations with clinical features. Eur Radiol 2007; 17(12): 3073-3078.

14. Riyazi N, Meulenbelt I, Kroon HM et al. Evidence for familial aggregation of hand, hip, and spine but not knee osteoarthritis in siblings with multiple joint involvement: the GARP study. Ann Rheum Dis 2005; 64(3): 438-443.

15. Kellgren JH, Lawrence JS. Radiological assessment of osteoarthrosis. Ann Rheum Dis 1957; 16(4): 494-502.

16. Altman R, Asch E, Bloch D, Bole G, et al. Development of criteria for the classification and reporting of osteoarthritis.
Classification of osteoarthritis of the knee. Diagnostic and Therapeutic Criteria Committee of the American Rheumatism Association. Arthritis Rheum 1986;29(8):1039-49.

17. Altman R, Alarcon G, Appelrouth D et al. The American College of Rheumatology criteria for the classification and reporting of osteoarthritis of the hip. Arthritis Rheum 1991; 34(5): 505-514.

18. Kothari M, Guermazi A, von Ingersleben $G$ et al. Fixed-flexion radiography of the knee provides reproducible joint space width measurements in osteoarthritis. Eur Radiol 2004; 14(9): 15681573.

19. Altman RD, Hochberg M, Murphy WA, Jr., Wolfe F, Lequesne M. Atlas of individual radiographic features in osteoarthritis. Osteoarthritis Cartilage 1995; 3(Suppl A): 3-70.

20. Altman RD, Gold GE. Atlas of individual radiographic features in osteoarthritis, revised. Osteoarthritis Cartilage 2007; 15(Suppl A): A1-56.

21. Kornaat PR, Watt I, Riyazi N, Kloppenburg M, Bloem JL. The relationship between the MRI features of mild osteoarthritis in the patellofemoral and tibiofemoral compartments of the knee. European Radiology 2005; 15(8): 1538-1543.

22. Yulish BS, Montanez J, Goodfellow DB, Bryan PJ, Mulopulos GP, Modic MT. Chondromalacia patellae: assessment with MR imaging. Radiology 1987; 164(3): 763-766.

23. Mink JH, Deutsch AL. Occult cartilage and bone injuries of the knee: detection, classification, and assessment with MR imaging. Radiology 1989; 170(3 Pt 1): 823-829.

24. Stoller DW, Martin C, Crues JV, III, Kaplan L, Mink JH. Meniscal tears: pathologic correlation with MR imaging. Radiology 1987; 163(3): 731-735.

25. McCauley TR, Kornaat PR, Jee WH. Central osteophytes in the knee: prevalence and association with cartilage defects on MR imaging. Am J Roentgenol 2001; 176(2): 359-364.

26. Zhang J, Yu KF. What's the relative risk? A method of correcting the odds ratio in cohort studies of common outcomes. JAMA 1998; 280(19): 1690-1691.

27. Hunter DJ, Zhang YQ, Tu X et al. Change in joint space width: hyaline articular cartilage loss or alteration in meniscus? Arthritis Rheum 2006; 54(8): 2488-2495.

28. Meister K, Indelicato PA, Spanier S, Franklin J, Batts J. Histology of the torn meniscus: a comparison of histologic differences in meniscal tissue between tears in anterior cruciate ligament-intact and anterior cruciate ligament-deficient knees. Am J Sports Med 2004; 32(6): 1479-1483.

29. Ding C, Martel-Pelletier J, Pelletier JP et al. Knee meniscal extrusion in a largely non-osteoarthritic cohort: association with greater loss of cartilage volume. Arthritis Res Ther 2007; 9(2): R21.

30. Lerer DB, Umans HR, Hu MX, Jones MH. The role of meniscal root pathology and radial meniscal tear in medial meniscal extrusion. Skeletal Radiol 2004; 33(10): 569-574.

31. Watrin A, Ruaud JP, Olivier PT et al. T2 mapping of rat patellar cartilage. Radiology 2001; 219(2): 395-402.

32. Hunter DJ, Zhang YQ, Niu JB et al. The association of meniscal pathologic changes with cartilage loss in symptomatic knee osteoarthritis. Arthritis Rheum 2006; 54(3): 795-801.

33. Felson DT, McLaughlin S, Goggins J et al. Bone marrow edema and its relation to progression of knee osteoarthritis. Ann Intern Med 2003; 139 (5 Pt 1): 330-336.

34. Hunter DJ, Zhang Y, Niu J et al. Increase in bone marrow lesions associated with cartilage loss: a longitudinal magnetic resonance imaging study of knee osteoarthritis. Arthritis Rheum 2006; 54(5): $1529-1535$. 